\title{
COMENTARIO A LA LEY 2/1986, DE 5 DE JUNIO, DE LA CULTURA FISICA Y DEL DEPORTE DE LA COMUNIDAD DE MADRID
}

\author{
por \\ Angel L. Monge Gil \\ Profesor Ayudante de Derecho Mercantil de la Universidad \\ de Zaragoza
}

SUMARIO: I. INTRODUCCION.-II. ASPECTOS GENERALES DE LA LEY DE LA CULTURA FISICA Y DEL DEPORTE DE LA COMUNIDAD DE MADRID. - III. CUESTIONES RELATIVAS AL ASOCIACIONISMO DEPORTIVO EN LA LEY DE LA CULTURA FISICA Y DEL DEPORTE DE LA COMUNIDAD DE MADRID.-IV. EL REGIMEN JURIDICO DEPORTIVO.

\section{INTRODUCCION}

La palabra que mejor define las relaciones existentes en nuestro País a lo largo de su historia más reciente entre el fenómeno deportivo y el mundo del Derecho es la ignorancia mutua. A esta lamentable situación no se le puede buscar un solo culpable. En efecto, si bien es cierto que el jurista no se ha acercado con demasiada asiduidad al deporte - gran parte de culpa de este desinterés la tiene la tan absurda como por otra parte extendida creencia de la dicotomía cabeza-músculo y de que el intelectual debía huir por principio de cuestiones que se desviasen de la más pura actividad del homo sapiens-, no resulta menos constatable el poco interés que ha mos- 
trado el mundo del deporte en adaptar sus estructuras a las normas de Derecho común -entendiendo por tal, en una concepción amplia del término, las normas generales del Derecho civil, administrativo, mercantil, penal, etc.-, debido, fundamentalmente, a un buen número de privilegios y prebendas de los que con demasiada frecuencia han gozado los dirigentes y rectores del deporte patrio, por lo que, en la medida de lo posible, se ha intentado dilatar unas situaciones que por muchos motivos se antojan ya insostenibles.

Buena prueba de lo anteriormente expuesto es que hasta el año 1961 en nuestro País, el deporte no tuvo una regulación plasmada en una norma con rango de ley, y cuando ésta surgió - la Ley sobre Educación Física de 23 de diciembre de 1961 - lo fue con finalidades que muchas veces traspasaban el campo de lo estrictamente deportivo, utilizándose éste como instrumento político de primera magnitud. (Esta idea se ve reflejada en el Preámbulo de la Ley General para la Cultura Física y Deporte de 31 de marzo de 1980, que a modo de matizado reconocimiento de culpas señala cómo «en España el progresivo intervencionismo público en el ámbito del deporte llevó a la aprobación de la Ley de Educación Física de 1961, que por primera vez se ocupa de la materia con visión amplia y alto rango normativo. A pesar de ser ésta una Ley profundamente innovadora [?], en cuanto habilitó importantes recursos económicos para el deporte con carácter permanente a nivel central y, también a escala local, adoleció de una estructura organizativa adecuada, debida a la escasa participación de los deportistas y a su explicable pero indebida vinculación a las instancias políticas imperantes en el momento de ser aprobada».) Pero es que con anterioridad a la publicación de la Ley de 1961 la situación era todavía más sangrante. Las normas que regularon nuestro deporte — normas que por cierto no fueron publicadas en los instrumentos oficiales del Estado- respondieron a una instrumentalización política llevada a cabo sin tapujos y puesta de manifiesto sin el más mínimo pudor.

Fue, según señala GoNZÁlez GRIMALDo, en el año 1941 «cuando al tomarse conciencia de la importancia política, social y económica se constituye la Delegación Nacional de esa actividad, quedando incardinada a la estructura orgánica de Falange Española» (1). Igualmente se exigía que, como manifestaba de forma inequívoca el artículo 42 del Estatuto Orgánico de la Delegación Nacional de Edu-

(1) GonzÁlez Grimaldo, Carmelo: El ordenamiento jurídico del deporte, Civitas, Madrid, 1974, pág. 25. 
cación Física y Deporte —creada por Decreto de 22 de febrero de 1941-, «los miembros de las Federaciones nacionales y los rectores de los clubs o sociedades deportivas habrán de tener una probada adhesión al Movimiento Nacional», castigándose con la descalificación, ex artículo 76 del propio Estatuto, a los deportistas que se dirigiesen a otra instancia que no fuese la Delegación Nacional para resolver asuntos disciplinarios.

Todas estas circunstancias conformaron la idea del deporte como fenómeno por una serie de intereses concurrentes ya expuestos, aislado dentro del entramado social existente, llegándose a lo que ha venido calificándose como «complejo de isla del fenómeno deportivo», así como a crear una situación que, en acertada calificación de BERMEJo VERA, podemos considerar como de «Derecho al margen del Derecho» (2).

La aprobación mediante referéndum por el pueblo español de la Ley para la Reforma Política de 4 de enero de 1977 incidió en gran medida en las estructuras del Movimiento Nacional.

Mediante el Real Decreto-ley de 1 de abril de 1977 se transfirieron a la Administración Pública todos los organismos del Movimiento que desarrollaban funciones de carácter social, incluyéndose entre éstos la promoción del deporte y de la cultura.

El Real Decreto-ley anteriormente citado contemplaba la creación de la Subsecretaría de Familia, Juventud y Deporte (art. 3.०), dependiente de Presidencia del Gobierno, como órgano encargado, entre otras cosas, de la preparación, dirección y ejecución de la política del Gobierno en lo referente a la educación física y a la práctica deportiva.

La Subsecretaría de Familia, Juventud y Deporte fue suprimida por Decreto de 29 de julio de 1977, procediéndose a la creación, por Decreto de 27 de agosto de 1977, de denominación, estructura y funciones del Ministerio de Cultura, del Organismo autónomo Consejo Superior de Deportes.

La Constitución española de 1978 proclama en su artículo 43, 3, el deber de fomento del deporte de todas las Administraciones Públicas, y así:

«Los poderes públicos fomentarán la educación sanitaria, la edu. cación física y el deporte. Asimismo, facilitarán la adecuada utilización del ocios.

(2) Bermejo Vera, José: «Asociacionismo deportivo», en la obra colectiva El De. recho deportivo, UNISPORT, Junta de Andalucía, Málaga, 1986. 
Dada esta extensión del deber de fomento del deporte, se hace imprescindible establecer cuál es el campo de actuación correspondiente a cada Administración Pública en concreto, a efectos de que el principio de eficacia proclamado en nuestra Carta magna (artículo 103,1$)$ como criterio de actuación de todas las Administraciones Públicas no quede en una mera declaración de principios a causa de una mala distribución de competencias.

En este sentido, y por lo que aquí nos interesa, el artículo 148 de la Constitución española, en sede de distribución de competencias Estado-Comunidades Autónomas, establece en el punto 19 que:

«Las Comunidades Autónomas podrán asumir competencias en las siguientes materias:

19. Promoción del deporte y de la adecuada utilización del ocio».

Dos conclusiones fundamentales, expuestas de forma sintética, se pueden extraer del contenido del artículo 148, 1, 19 de la Constitución española.

La primera de ellas es que, desde la perspectiva de las Comunidades Autónomas, todas ellas pueden - recalquemos el matiz potestativo- asumir competencias en materia deportiva, siempre y cuando así aparezca recogido en sus respectivos Estatutos de Autonomía -norma constitucional básica en cada Comunidad Autónoma, dentro de los términos de la Constitución española de 1978, ex art. 147, 1, de ésta- y que podrán ampliar sus competencias transcurridos cinco años desde la aprobación de sus Estatutos, respetando unos mínimos que correspondan al Estado (3), teniendo en cuenta que las Comunidades Autónomas calificadas como de «primer grado» - configuración conforme al art. $151 \mathrm{CE}$ y Disposición transitoria segunda de la misma- no necesitan esperar esos cinco años para asumir competencias no exclusivas del Estado (4).

(3) Estos mínimos hay que justificarlos en aras del interés general existente en que una serie de funciones permanezcan siempre en la órbita del Estado.

En opinión de Cazorla Prieto estas funciones serían:

1. Coordinación.

2. Organización de acontecimientos deportivos de importancia nacional o internacional.

3. Armonización, planificación y representación internacional del deporte nacional.

Vid. Cazorla Prieto, Luis-María: Deporte y Estado, Labor Politeia, Barcelona, 1979, pág. 191.

(4) Sobre el tema del acceso a la autonomía y sus modalidades, vid., por todos, 
La segunda conclusión a la que se hacía referencia viene referida a la consideración de la materia deportiva como una competencia no atribuible de forma exclusiva ni al Estado ni a las Comunidades Autónomas, sino que será de aquellas calificables como compartidas o concurrentes.

Así pues, y recalcamos la idea, para que una Comunidad Autónoma pueda asumir competencias en materia deportiva es necesario que, en primer lugar, en su respectivo Estatuto de Autonomía venga contemplada tal posibilidad.

En el Boletín Oficial del Estado de 11 de octubre de 1986 apareció publicada la Ley $2 / 1986$, de 5 de junio, de la Cultura Física y del Deporte de la Comunidad de Madrid, desarrollando así la competencia recogida en el artículo 26, 17, de la Ley Orgánica 3/1983, de 25 de febrero, por la que se aprobó el Estatuto de Autonomía de esta Comunidad Autónoma, y que reconoce a la misma la competencia exclusiva - téngase en cuenta el alcance que hay que dar a esta declaración de exclusividad, tal y como se expuso anteriormente (5)- en materia de promoción del deporte.

La Ley de 5 de junio de 1986 tiene el mérito fundamental de haber abierto el fuego, con todos los riesgos que ello conlleva, en la batería legislativa que previsiblemente en un corto espacio de tiempo va a emanar de los órganos legislativos de las Comunidades Autónomas por lo que a la materia deportiva se refiere, en cumplimiento de las previsiones de sus respectivos Estatutos de Autonomia.

En la Exposición de motivos de la Ley objeto de este comentario se recoge una afirmación que no deja de producir un cierto desasosiego, invitando, simultáneamente, a una serena reflexión. En concreto, nos referimos a las siguientes aseveraciones: «Pese a esta clara asignación de competencias fijadas en textos legales - refiriéndose a la Constitución española, Estatutos de Autonomía y Ley General de la Cultura Física y del Deporte-, existe una cierta con-

Muñoz Machado, Santiago: Derecho Público de las Comunidades Autonomas, tomo I, Civitas, Madrid, 1982, págs. 245-312.

(5) Pienso que las competencias que asuma una Comunidad Autónoma en aplicación de lo regulado en el artículo 148 no abarcarán todas las posibles funciones subsumibles dentro de una materia, sino que la Constitución lo único que contempla es que recaben funciones dentro de una serie de materias, pero no lo hagan sobre una materia en bloque (paradójicamente la única competencia que la Constitución reconoce a algunas Comunidades Autónomas con carácter de exclusividad estaría dentro de las materias recogidas en el artículo 149, en principio reservadas a la competencia del Estado, y vendría referida a los derechos civiles forales o especiales allí donde existan). 
fusión y sensación de provisionalidad hasta que una normativa ajuste adecuadamente esta cuestión».

Del tenor literal de las afirmaciones ahora transcritas parece ser que desde esta Ley autónoma se está invocando la promulgación de una nueva Ley General del Deporte en nuestro País -emanada de la Administración central- que venga a paliar esas deficiencias de que, según la Comunidad de Madrid, adolece nuestro deporte, como un prius a la producción legislativa por parte de las Comunidades Autónomas, en el tema que nos ocupa.

Sea como fuere, lo cierto es que, por acontecimientos que están en la mente de todos, nuestro País goza de una ocasión histórica difícilmente repetible para proceder a una reestructuración de alguno de los pilares básicos sobre los que se sustenta el deporte en nuestra Nación, y en esta tarea las Comunidades Autónomas, al igual que el resto de los Poderes públicos, tienen mucho que decir.

En esta línea es en la que, a no dudarlo, hay que enmarcar la Ley de Cultura Física y del Deporte de la Comunidad de Madrid. No obstante esta reiterada valoración positiva de conjunto de la Ley, queremos comentar algunas cuestiones y plantear algunas dudas surgidas al hilo de una atenta lectura de la misma.

\section{ASPECTOS GENERALES DE LA LEY DE CULTURA FISICA Y DEL DEPORTE DE LA COMUNIDAD DE MADRID}

En primer lugar, y por lo que hace referencia al objeto de la Ley de 5 de junio de 1986, sorprenden algunas circunstancias. A lo largo de la Exposición de motivos de la misma el legislador marcó algunos objetivos muy amplios -objetivos que por cierto demuestran una finura jurídica bastante acusada al establecer como, en otros, objetivo general de la Ley ordenar la situación y funciones de los diferentes agentes del fenómeno deportivo, mientras que es un objetivo concreto de la misma ordenar la actuación de los diversos agentes del fenómeno deportivo-, los cuales aparecen sensiblemente reducidos en el tenor del artículo $10^{\circ}$, el cual establece que: «Es objetivo del deporte en el ámbito de la Comunidad de Madrid, tanto en lo relativo a la tutela y promoción de las actividades organizadas por el sector privado como el fomento y coordinación de las iniciativas públicas y las relaciones entre un sector y otro".

Es opinión de nuestro Tribunal Constitucional que los Preám- 
bulos tienen valor meramente interpretativo del articulado y que, por lo tanto, a la hora de delimitar el objeto de la Ley de la Cultura Física y del Deporte de la Comunidad de Madrid se debe atender al contenido del artículo $1 .^{\circ}$

Aun cuando en el propio artículo $10^{\circ}$ parece establecerse una actitud diferente por parte de la Comunidad Autónoma de Madrid según las actividades deportivas sean organizadas por el sector privado - promoción - o el sector público - fomento-, en realidad esta pretendida dicotomía no existe. La actividad de fomento del deporte, de la forma en que viene recogida en el artículo 43, 3, de la Constitución española - referida, no se olvide, a todas las Administraciones Públicas - , consiste, en palabras de CAzorLA, en promover, proteger, financiar e impulsar el fenómeno deportivo (6). En consecuencia, la referencia realizada en el Estatuto de Autonomía de Madrid - al igual que en otros muchos Estatutos de Autonomía- a la competencia exclusiva de ésta en lo referente a promoción del deporte hace necesarias dos precisiones. En primer lugar, debe tenerse en cuenta la interpretación dada anteriormente al término exclusiva, en el sentido de hallarnos ante una competencia compartida o concurrente. En segundo lugar, que cuando se habla de competencia sobre la promoción del deporte sin duda se está pensando en el fomento en su totalidad, si bien se está designando el todo -el fomento- a través de una parte - la promoción- (7).

Finalmente, por lo que respecta al contenido del artículo $1 .^{\circ}$, se echa en falta una declaración expresa del derecho de los ciudadanos de la Comunidad de Madrid al conocimiento y práctica del deporte, de la forma que se realiza en la Ley General de la Cultura Física y del Deporte de 31 de marzo de 1980 (BOE 12 abril), en su artículo $1 .^{\circ}$

Tampoco queda suficientemente claro en la Ley cuál es la actitud de la Comunidad Autónoma de Madrid con respecto al fenómeno deportivo. Varios son los adjetivos empleados por el legislador, y no siempre resultan armonizables. Así se pueden constatar actitudes que van desde la simple coordinación «... coordinación de las iniciativas públicas...» (art. 1., 1), hasta el control «... controlar la

(6) Cazorla Prieto, Luis-María: Op. cit., pág. 181.

(7) De hecho, las Comunidades Autónomas desde un principio han adoptado una actitud de fomento - con todo lo que ello conlleva - ante el fenómeno deportivo, lo que puede ser constatado analizando el contenido de los derechos de transferencias en materia de cultura.

Vid., por lo que al caso hace referencia, el Real Decreto 653/1985, de 19 de abril, sobre traspaso de funciones y servicios de la Administración del Estado a la Comunidad de Madrid en materia de deportes ( $B O E, 11$ mayo). 
eficacia de los presupuestos públicos dedicados a fomentar esas actividades..." (Exposición de motivos, arts. 15 y 29), pasando por la tutela (art. 1..$^{\circ}$ o la ordenación (Exposición de motivos).

La postura a adoptar por parte de los Poderes públicos al respecto se nos antoja trascendente y debe quedar perfectamente clara, pues de ella va a depender en buena medida la estructuración que del deporte se haga en un determinado ámbito territorial.

La evolución que en nuestro País se ha producido, por lo que respecta a la actitud de los Poderes públicos ante el fenómeno deportivo, es significativa. En un primer momento los Poderes públicos se mantuvieron esencialmente al margen, tal y como se afirma en el Preámbulo de la Ley General de la Cultura Física y del Deporte: "La actitud deportiva se ha venido produciendo en la época contemporánea como manifestación de iniciativas sociales espontáneas, al principio libres de todo intervencionismo por parte de los Poderes públicos, sin perjuicio de su natural sujeción a medidas de policía general...»

En una segunda etapa se puede hablar de intervencionismo de los Poderes públicos ante el fenómeno deportivo, debido, en palabras del propio Preámbulo de la LGCFD, al «crecimiento de la importancia del deporte en términos cuantitativos y cualitativos, su conexión con la problemática de la educación física al servicio del pleno desarrollo de la persona y su enorme trascendencia para la calidad de vida como objetivo de todo Estado social de Derecho...»

Por último, se puede vislumbrar un nuevo grado de intervencionismo mucho más acusado por parte de los Poderes públicos.

Buena prueba de ello la encontramos en las siguientes afirmaciones vertidas, por nuestro Tribunal Constitucional en la Sentencia de 10 de enero de 1986 (BOE 12 febrero), que resuelve el conflicto positivo de competencia promovido por el Consejo Ejecutivo de la Generalidad de Cataluña contra el artículo $4 .^{\circ}$, por conexión con los artículos $1 .^{\circ}, 3 .^{\circ}$ y $5 .^{\circ}$ del Real Decreto 2075/1982, de 9 de julio, sobre normas de actividades y representaciones deportivas internacionales. En su Fundamento jurídico primero afirma que:

«El segundo examen - aunque principal ya en el proceso conflictual- habrá de orientarse a determinar si, admitida en algún caso aquella participación, la eventual intervención pública en orden a su control corresponde, sobre las Federaciones catalanas, a la Administración deportiva de la Comunidad Autónoma, como ésta 
pretende, o habrá de atenderse, más bien, al régimen estatal de autorización al que remite el artículo del Real Decreto 2075/1982 .

$Y$ en el Fundamento jurídico tercero se añade que:

aSi no fuera así, por lo demás, se quebraría la coherencia y el sentido mismo de la intervención administrativa controvertida, en la que ha de buscarse, sin perjuicio de otras consideraciones no pertinentes ahora, que sean conexos los ámbitos de actuación del Ente controlado y de aquél que ejerce el control».

Estimamos que las Administraciones Públicas no deben caer en el error de adoptar ante el deporte actitudes - fundamentalmentefiscalizadoras. Una cosa es el intervencionismo de las Administraciones Públicas, por otra parte absolutamente deseable, dadas las funciones que el deporte cumple en nuestra sociedad, y otra cosa muy discutible es que éste deje paso al referido control. Parecen perfectamente trasladables a España las puntualizaciones realizadas por CHARLES con respecto a nuestro País vecino: «La organización del deporte en Francia reposa, en efecto, en un sector público y sobre un sector privado. No están separados, sino que están estrechamente unidos y complementados; ellos colaboran en una misión común, incluso si el sector privado depende del público» (8).

Es de lamentar, finalmente, que a lo largo de la Ley de la Comunidad Autónoma madrileña se caiga en dos de los vicios que más han marcado las estructuras deportivas en nuestro País, a saber, el denominado "complejo de isla" del fenómeno deportivo y la desconfianza de la Administración hacia los agentes deportivos.

En este sentido pueden leerse las siguientes afirmaciones contenidas en la Exposición de motivos: «Por otra parte, se hace imprescindible la protección de los agentes del fenómeno deportivo frente a diversos intereses para garantizar su independencia o alejar el peligro de indefensión que en algunas circunstancias puede darse»; y unos párrafos más adelante, y en esta misma línea, se señala cómo "conscientes de que resulta imprescindible la protección de los agentes del sistema deportivo frente a posibles interferencias ajenas al mismo, la Ley propone agilizar el funcionamiento de los órganos que imparten el régimen jurídico deportivo...» Flaco favor se hace al deporte español con ideas como las ahora reflejadas. Tan

(8) Charles, Hubert: «Les relations du groupement sportif et de la puissance publiquen, en el volumen colectivo Les problemes juridiques du sport. Le sportif et le groupement sportif, Centre d’Etudes Administratives, París, 1971, pág. 222. 
necesario e imprescindible resulta el sometimiento del deporte a las normas del Derecho común - con el alcance dado anteriormente al término- como que cese la tan absurda como reiterada desconfianza de la Administración hacia los agentes del sistema deportivo.

\section{CUESTIONES RELATIVAS AL ASOCIACIONISMO DEPORTIVO EN LA LEY DE LA CULTURA FISICA Y DEL DEPORTE DE LA COMUNIDAD DE MADRID}

Uno de los pilares clave sobre el que se sustenta el entramado. deportivo de nuestro País es, sin duda, el del asociacionismo deportivo. La tendencia natural del hombre de asociarse para desarrollar algún tipo de actividad alcanza también de forma manifiesta al deporte, con la peculiaridad de que el asociacionismo deportivo tiene una serie de notas que lo diferencian de los postulados esenciales del Derecho de asociación, el cual, por cierto, encuentra su más alta regulación normativa en el tenor del artículo 22 de la Constitución española (9).

Este régimen especial consiste, muy sintéticamente, en la posibilidad de asociarse al margen de los postulados de la Ley de Asociación de 1964, sometiéndose a los dictados de la Ley General de la Cultura Física y del Deporte, la cual ofrece una serie de ventajas a aquellas asociaciones que optan por este sistema, esencialmente la participación en competiciones oficiales y la entrega de subvenciones para cumplir una serie de fines preestablecidos, exigiéndose como contrapartida el cumplimiento de unos requisitos contemplados en la propia Ley General de la Cultura Física y del Deporte -aprobación de los Estatutos de los clubs deportivos por el Consejo Superior de Deportes y la inscripción en el Registro de Asocia-

(9) En el Preámbulo de la Ley General de la Cultura Física y del Deporte se justifica la existencia de un régimen asociativo especial con los siguientes argumentos:

«... el crecimiento de la importancia del deporte en términos cuantitativos y cualitativos, su conexión con la problemática de la educación física al servicio del pleno desarrollo de la persona y su enorme trascendencia para la calidad de vida como objetivo de todo Estado social de Derecho, no sólo en el plano nacional, sino también en el internacional, donde contribuye a incrementar y fortalecer las relaciones y entendimiento entre los pueblos, son factores que, sin duda, han llevado a los poderes públicos a ocuparse del deporte... hasta el punto de originar un régimen asociativo o introducir importantes singularidades en el régimen jurídico general de las asociaciones... Es preciso resaltar que se establece en la Ley un régimen asociativo especial para las asociaciones que tienen como finalidad específica la educación física y el deporte». 
ciones Deportivas para alcanzar el reconocimiento legal a efectos de la Ley-. Y todo ello sin perjuicio de que existan asociaciones deportivas constituidas conforme a la Ley de Asociación de 1964 y no a la Ley General de la Cultura Física y del Deporte.

El Tribunal Constitucional ha tenido ocasión de pronunciarse sobre la cuestión en la Sentencia de 24 de mayo de 1985 (BOE 27 junio) (10), declarando en su Fundamento jurídico tercero, por lo que aquí nos interesa, que en el caso de las asociaciones deportivas:

"... nos hallamos ante un supuesto de las denominadas de aconfiguración legals reguladas, siempre teniendo en cuenta los límites del artículo 22, al margen de la Ley de Asociaciones de 1964 justificándolo en el hecho de que el mencionado precepto no comprende el derecho de constituir asociaciones para el ejercicio de funciones públicas de carácter administrativo relativos a un sector de la vida socialn.

Pues bien: hechas estas breves puntualizaciones en torno al asociacionismo deportivo, son varias las interrogantes que sobre la cuestión nos planteamos.

La primera de ellas es la de si en la Comunidad Autónoma de Madrid, frente a lo que sucede a nivel nacional en donde existe un único Registro de Asociaciones Deportivas (art. 12, 1, LGCFD), existen dos Registros diferentes que cumplen las mismas funciones que éste en el seno de la Comunidad Autónoma.

La duda surge porque en el artículo $4 .^{\circ}, 1$, de la Ley madrileña, artículo encuadrado en la sección primera del capítulo II y que lleva por título "Normas comunes sobre asociacionismo deportivo", se dice que:

«A fin de acceder a los derechos y beneficios previstos en la pre. sente Ley, las Asociaciones y Agrupaciones a que se refiere el artículo anterior deberán solicitar su inscripción en el Registro de Entidades Deportivas de la Comunidad de Madrid...».

Por contra, en el artículo 12, 2, dentro de la sección segunda del propio capítulo II («De los diversos tipos de Entidades deportivas»), se dispone que:

«Las Agrupaciones deportivas podrán participar en las competiciones oficiales federadas, si así lo desean. En tal caso, y exclusivamente a estos efectos, deberán solicitar su inscripción en el Registro de Asociaciones Deportivas».

(10) Vid. el comentario que de la Sentencia citada realiza Esteve Pardo en REDA, número 35 , págs. 113-121. 
$¿$ Nos hallamos en presencia de dos Registros diferentes con funciones idénticas? ¿Estamos ante dos Registros diferentes con funciones igualmente diferentes?

Parece que el sentido común inclina a pensar que en la Comunidad Autónoma de Madrid existe un único Registro de Asociaciones Deportivas con competencias en el territorio de la Comunidad Autónoma y que simplemente cabe achacar la disparidad de denominaciones a un ligero descuido del legislador, por otra parte sin mayor trascendencia.

Mayores problemas de legalidad pueden presentar algunas afirmaciones vertidas en el curso del articulado de la Ley en lo referente a libertad de asociación, libertad reconocida, no se olvide, al más alto rango normativo (art. $22 \mathrm{CE}$ ).

En el artículo $6 .^{\circ}, 1$, se establece con carácter taxativo que:

«Todos los miembros de las Entidades deportivas que agrupen exclusivamente a personas físicas, cualquiera que sea la finalidad, nivel de prácticas realizadas o sistema de financiación, deberán tener la condición de sociosn.

¿Es correcta la exigencia de tener la condición de socio para poder ser miembro de una Entidad deportiva? Tal exigencia, ¿conculcaría en alguna medida el artículo 22 de la Constitución española?

Con respecto a este punto hay que tener en cuenta que dentro de una Entidad deportiva no existe una sola categoría de miembros. Ello resulta de enorme importancia en una cuestión que desgraciadamente está de indudable actualidad. Nos estamos refiriendo a la responsabilidad de los diferentes miembros de las Entidades deportivas por las deudas acumuladas por éstas. No todos los miembros responderán de igual forma, porque los derechos y deberes tampoco son los mismos. En una Entidad deportiva se pueden distinguir, esencialmente, entre socios y abonados. La diferencia entre ambos es sustancial. Así, mientras que los abonados serían aquellos miembros que solamente pagan una cantidad de dinero a cambio de poder asistir a un número determinado de espectáculos deportivos sin tener un animus cierto de formar parte de la «masa social» del club, los socios serían aquellos que voluntaria y conscientemente deciden integrarse en esa masa social, de lo cual se van a derivar una serie de derechos y obligaciones consustanciales a su condición de socios. En el caso de los abonados, su responsabilidad se circunscribe estrictamente a lo que es el pago de una cantidad 
-bien de una vez, bien fraccionada- determinada previamente por el club, sin que se les pueda exigir ninguna otra obligación -aparte de la de mantener un correcto comportamiento dentro de los recintos e instalaciones deportivas-, ni responderán de las posibles deudas del club con más cantidad de la ya aportada en concepto de abono.

Por contra, la situación de los socios es bien distinta. Son los verdaderos propietarios del club, y como tales deben responder, conforme a las normas previstas en lo tocante a la responsabilidad de la sociedad civil - de cara al exterior-y a la comunidad de bienes -en el ámbito de las relaciones entre socios-.

Por todo ello no parece correcto el contenido del artículo $6 .^{\circ}$, ya que supone una más que discreta injerencia en la libertad de asociarse a una Entidad deportiva por parte de un ciudadano, el cual puede preferir mantener, por los motivos antes señalados, una vinculación diferente a la de socio, como pretende la Ley madrileña.

Por otra parte, y en relación con esta misma cuestión, sorprende la referencia realizada en el artículo $27, b$ ), de la Ley, que, refiriéndose al régimen económico de las Entidades deportivas, señala que:

«Podrán fomentar manifestaciones de carácter físico-deportivo dirigidas al público en general, aplicando los beneficios obtenidos al desarrollo de actividades físicas y deportivas para sus asociados».

Sorprende, insistimos, la declaración tajante contenida en el artículo $6 .^{\circ}$, por una parte, y, por otra, la utilización de otros sustantivos que pueden inducir, al menos, a algún tipo de confusión.

Una nueva duda con respecto a una posible violación del artículo 22 de la Constitución española se suscita por la declaración del artículo $7 .^{\circ}, 3$, de la Ley, que señala que:

"La Comunidad de Madrid regulará reglamentariamente las competencias de los órganos de gobierno y representación de disciplina deportiva y los demás aspectos relativos al desarrollo de las actividades de las Asociaciones deportivas».

Insistimos que nos parece al menos dudoso que la Administración pueda, por vía reglamentaria, incidir en aspectos puramente organizativos de unas asociaciones, en este caso deportivas. Las cuestiones contempladas en el artículo $7 .^{\circ}, 3$, pensamos que deban quedar dentro del buen criterio de cada asociación, siempre, eso sí, 
respetando el contenido del artículo 22 de la Constitución española (11).

También parece discutible, o al menos precisa de alguna aclaración, el tenor de la Disposición transitoria primera de la Ley, en virtud de la cual:

«Las Asociaciones deportivas radicadas en el territorio de la Comunidad de Madrid, en el plazo de un año a partir de la entrada en vigor de la reglamentación sobre Registro de Entidades Deportivas prevista en esta Ley, habrán de adaptar sus Estatutos a la misma y solicitar su inscripción en el mencionado Registro».

Lógicamente, aunque la Ley no realice la diferenciación, el contenido de esta Disposición transitoria sólo será de aplicación a aquellas asociaciones deportivas constituidas conforme a la Ley de la Cultura Física y del Deporte y no a aquellas que hayan optado por constituirse conforme a las exigencias de la Ley de Asociación de 1964.

Un segundo gran apartado dentro de este punto dedicado al asociacionismo deportivo en la Ley de la Cultura Física y del Deporte de la Comunidad madrileña, vamos a dedicarlo a la regulación que en la misma se realiza de las Federaciones territoriales en tanto que Entes asociativos de "segundo grado" - considerando a los clubs y agrupaciones deportivas como Entes asociativos de "primer gradow- de naturaleza, tal y como ha subrayado el propio Tribunal Constitucional en la ya citada Sentencia de 24 de mayo de 1985, privada, pero a las que se atribuyen funciones públicas de carácter administrativo (Fundamento jurídico cuarto).

No parece muy acertado, en primer lugar, el papel que la Ley madrileña atribuye a las Federaciones territoriales.

Tras realizarse a lo largo de la Exposición de motivos una severa crítica del papel y cometidos desempeñados por las Federaciones territoriales, lamentándose de cómo «la función de coordinación, característica de cualquier Federación allende de nuestras

(11) A nivel nacional existe el precedente del Real Decreto $177 / 1981$, de 16 de enero, del Ministerio de Cultura, sobre normas reguladoras de clubs y Federaciones que contempla hasta el más mínimo detalle la forma de organización de éstos.

El Club Deportivo Hispano-Francés y otros solicitaron que fuese declarado no conforme a Derecho y, por tanto, nulo, al estimar que todos los preceptos de los capítulos $10^{\circ}$ y $2^{\circ}$ del referido Real Decreto vulneraban el contenido del artículo $22 \mathrm{CE}$.

EI Tribunal Supremo conoció del asunto en la Sentencia de 10 de febrero de 1984 (Aranzadi 797), fallado en el sentido de no admitir el recurso al fallar el requisito previo de la interposición del recurso de reposición con carácter previo, por lo que; lamentablemente, no entró a conocer sobre el fondo del asunto. 
fronteras, dejó lugar prioritario a la acción de fomento y extensión por la endeblez tradicional de nuestro entramado deportivo", se expresa a continuación que el papel que se asigna a las Federaciones territoriales madrileñas es el de «lograr la coordinación y enlace del deporte madrileño con el nacional».

A este respecto consideramos oportuno recordar que el fomento del deporte por parte de los Poderes públicos es un mandato constitucional (art. 43,3) y que esta tarea puede ser llevada a cabo tanto de una forma directa como de otra indirecta, a través de las Federaciones deportivas, y que el papel de las Federaciones territoriales no puede residir en la realización de funciones de coordinación y enlace con las respectivas Federaciones nacionales, ya que de ser así se convertirian en unos meros órganos gestores con muy poco campo de acción.

Una novedad importante, y en principio digna de las mayores loas, de la Ley madrileña con respecto a las disposiciones de la Ley General de la Cultura Física y del Deporte estriba en el mecanismo de constitución de una Federación deportiva. El artículo 11, 3, de la Ley madrileña dispone que:

«La Comunidad de Madrid, a la vista de las solicitudes existentes, podrá reconocer libremente la que estime más conveniente, atendiendo a criterios de implicación efectiva, racionalidad y de la mejor organización de la estructura deportiva en el territorio de Madrid, previo informe del Consejo Regional de Deportes».

La idea, repetimos, en principio es buena; pero, por los motivos que a continuación se expondrán, parece no puede llevarse a efecto.

En el fondo de la cuestión pensamos que subyace el caso de la Federación (provisional) Española de Fútbol-Sala, inscrita -tras muchos avatares e incluso resoluciones judiciales contradictoriasen el Registro de Asociaciones y Federaciones Deportivas, en cumplimiento de un Auto de la Audiencia Nacional de 22 de enero de 1986, que obligaba al Consejo Superior de Deportes a esta inscripción en cumplimiento de la Sentencia de 3 de abril de 1985.

No obstante, sostenemos la inviabilidad de lo dispuesto en el artículo 11,3 , de la Ley madrileña. Las razones que nos llevan a tan tajante conclusión son las expuestas por la Audiencia Nacional en la ya citada Sentencia de 3 de abril de 1985, la cual sostiene que «... interpretando a la luz de la Constitución española la Ley 13/ 1980, de 31 de marzo, sobre Cultura Física y el Deporte, y el Real 
Decreto $177 / 1981$, de 16 de enero, se establece que para el pleno ejercicio del derecho de asociación reconocido en el artículo 22 de la Constitución, la inscripción en el Registro sólo podría denegarse para impedir el acceso al mismo de aquellas específicamente aludidas en los números 2 y 5 , lo que, a sensu contrario, equivale a establecer que la inscripción resulta obligada en aquellos supuestos de asociaciones cuyos lícitos fines no hayan sido puestos en duda o lo. sean sin fundamento, y si ello resulta aplicable a las asociaciones en general, con mucha mayor razón lo serán cuando se trate de asociaciones deportivas...»

Por ello, y al recogerse conforme en la Ley que sólo puede existir una Federación regional (?) para cada especialidad deportiva (artículo 11,4$)$, la posibilidad de llevar a la práctica el contenido del artículo 11, 3, es improbable (12).

Lo que no parece en modo alguno sostenible es el contenido del artículo 27, e), referido a la disolución de las Federaciones regionales. El citado precepto establece que:

«En el caso de disolución de una Federación regional, el destino de sus bienes lo decidirá la Consejería de Cultura y Deportes».

Una cosa es que, dadas las tareas de promoción del deporte que cumplen las Federaciones deportivas, en caso de disolución de éstas. sus bienes reviertan a la colectividad, pero, eso sí, conforme a una normativa preestablecida - como se contempla en el art. 18, $d$ ), de la Ley General de la Cultura Física y del Deporte, que remite a las. disposiciones civiles y administrativas sobre la materia-, y otra. cosa es que una norma con rango de ley abra la puerta a una posible arbitrariedad a la hora de decidir el destino de los bienes de una Federación territorial. Esos bienes cumplen una misión importante, y ésta debe seguirse cumpliendo en caso de disolución de la Federación correspondiente.

(12) La solución, tal vez, puede ir en el sentido de que se permitiese la existencia de varias Federaciones por cada especialidad deportiva, pero que de ellas solamente una fuese la oficial, la cual sería designada por la Administración, siendo los Tribunales Contencioso-Administrativos los que en caso de reiterado y grave incumplimiento. de las funciones públicas autorizasen a la Administración para dotar a otra Federación. con el carácter oficial y, por tanto, pasando a disfrutar de las ventajas ofrecidas por aquélla. 


\section{EL REGIMEN JURIDICO DEPORTIVO}

El capítulo V de la Ley lleva por título «Del régimen jurídico deportivo", y en el mismo se contienen tres secciones dedicadas a la Comisión Jurídica del Deporte, al recurso administrativo disciplinario y al recurso administrativo electoral, respectivamente.

Lo primero que sorprende de este capítulo es el propio título del mismo.

¿Es que acaso no se puede aplicar el calificativo de «jurídico» para el resto de la Ley? ¿Sólo es «jurídico» lo contenido en el capítulo V? Y, en tal supuesto, ¿qué calificación merece el resto de la Ley de la Cultura Física y del Deporte de la Comunidad de Madrid?

La explicación que sospechamos puede dar respuesta a tan insólita situación, tal vez haya que buscarla en la tradicional creencia popular de que sólo es "jurídico" y merece tal calificación aquello que se identifica con la sanción, con el castigo o la pena. $Y$, en efecto, examinando el contenido del capítulo $\mathrm{V}$, no se nos ocurre otra explicación mejor.

Por lo que respecta a la regulación de la Comisión Jurídica del Deporte, sorprenden dos circunstancias. En primer lugar, la parca atención que se dedica a lo que son y en qué consisten las competencias de la Comisión, ya que la Ley dedica inexplicablemente casi toda su atención en fijar las causas y procedimientos a seguir para suspender a los miembros de la Comisión del ejercicio de su cargo.

En segundo término, la composición de la Comisión cabe calificarla de curiosa. En efecto, el artículo 30 , 2, señala que:

«La Comisión estará integrada por cinco miembros elegidos pre. ferentemente entre Licenciados en Derecho de reconocida experiencia en Derecho deportivo...».

El vocablo que en nuestra opinión resulta absolutamente distorsionador y que puede llegar a producir resultados rayantes con la hilaridad es el adverbio preferentemente. Con él se puede llegar a legitimar no sólo que algunos de los miembros de la Comisión no tengan experiencia en Derecho deportivo, sino que, y esto es lo más grave, que alguno de sus miembros ni siquiera sea licenciado en Derecho. 
Una vez finalizado este breve comentario a alguna de las cuestiones que nos ha sugerido la lectura de la Ley de la Cultura Física y del Deporte de la Comunidad madrileña, comentario realizado, por otra parte, sin pretender abarcar todos y cada uno de los sugestivos temas que en la misma se contemplan, nos resta desear que las futuras Leyes del Deporte de las Comunidades Autónomas, que previsiblemente irán viendo la luz en un futuro no muy lejano, sirvan para proceder a una mejor reestructuración del deporte en nuestro País, en la línea en la que se sitúa, reiteremos, la Ley madrileña del Deporte, objeto de este comentario. 


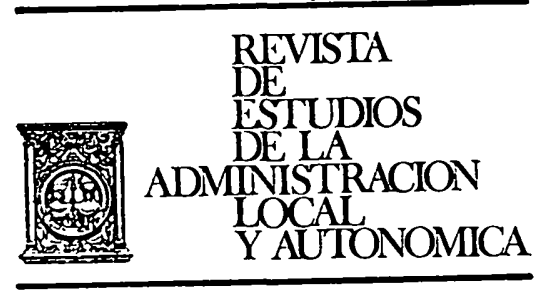

\section{JURISPRUDENCIA}


REALA-1986, núm. 232. MONGE GIL, ANGEL L.. COMENTARIO A LA LEY 2/1986, DE 5 DE J...

REALA-1986, núm. 232. MONGE GIL, ANGEL L.. COMENTARIO A LA LEY 2/1986, DE 5 DE J... 